\title{
Book review: Kritische Geographien ländlicher Entwicklung. Globale Transformationen und lokale Herausforderungen
}

\author{
Falk Künstler \\ Institut für Humangeographie, Goethe-Universität Frankfurt am Main, \\ Theodor-W.-Adorno-Platz 6, 60629 Frankfurt am Main, Deutschland \\ Correspondence: Falk Künstler (falk.kuenstler@stud.uni-frankfurt.de) \\ Published: 8 April 2020
}

\begin{abstract}
Mießner, M. und Naumann, M. (Hrsg.): Kritische Geographien ländlicher Entwicklung. Globale Transformationen und lokale Herausforderungen, Verlag Westfälisches Dampfboot, Münster, 296 pp., ISBN 978-3-89691-277-0, EUR 33,00, 2019.
\end{abstract}

In ihrer Einleitung zu diesem Sammelband, der 2019 in der Buchreihe „Raumproduktionen: Theorie und Gesellschaftliche Praxis“ erschienen ist, weisen Michael Mießner und Matthias Naumann auf das Spannungsfeld zwischen bestehenden Strukturproblemen ländlicher Räume und dem politischen Bekenntnis zur Berücksichtigung ländlicher Entwicklung hin. Obwohl ein Großteil der Menschen in Deutschland in kleinen und mittelgroßen Städten sowie Dörfern lebt, werden viele gesellschaftliche Entwicklungen als städtische Phänomene diskutiert. Dies gilt für wissenschaftliche Fragestellungen, aber auch bei Akteuren aus Politik, Planung und Zivilgesellschaft scheint in Bezug auf zielführende und progressive Interventionen im ländlichen Raum meist Ratlosigkeit vorzuherrschen. Wenn ländliche Räume in den Fokus politischer und medialer Aufmerksamkeit geraten, dann meist mit Blick auf den drastischen Rückbau von öffentlicher Infrastruktur, hoch verschuldete Kommunen oder die konstatierte „Landflucht“ in Ballungsgebiete. Ländliche Gebiete rivalisieren mit ökonomisch leistungsfähigeren Städten um den Bezug von Strukturförderungen und erhalten diese vornehmlich nur an neoliberale Spar- und Reformauflagen geknüpft. Gleichzeitig stilisieren sich rechtspopulistische Parteien und Bewegungen zu Rettern einer vernachlässigten ländlichen Bevölkerung und instrumentalisieren sozioökonomische Disparitäten für ihre politischen Ziele.
Es wird deutlich, dass der Bedarf an gesellschaftskritischer Forschung zum ländlichen Raum immens ist. Die 19 Beiträge dieser Publikation stoßen mit unterschiedlichen Fragestellungen in ein bislang kaum besetztes Forschungsfeld vor. Dabei zeigen die Autor*innen auf, wie gerade die englischsprachige Debatte als Anknüpfungspunkt dienen kann. Denn hier hat sich die Rural Geography als eigener Forschungsstrang etabliert, der in Teilen eine dezidiert kritische Perspektive aufweist. Der Sammelband will theoretische Perspektiven präsentieren, auf deren Grundlage sich eine Kritische Geographie ländlicher Räume entwickeln lässt und überdies aktuelle Forschungsprojekte, die ihren Blick weit über den deutschsprachigen Raum hinaus richten. Einige Schwerpunkte der Publikation werden im Folgenden kurz vorgestellt.

$\mathrm{Zu}$ Beginn skizziert der Band zentrale Entwicklungswicklungslinien einer Kritischen Geographie des ländlichen Raums und bietet so einen fundierten thematischen Einstieg. Florian Dünckmann untersucht wie „das Ländliche“ diskursiv produziert und beschrieben wird. Ländliche Räume werden danach oftmals als ,rückständige Resträume“ oder als romantisierte Idyllen (Authentizität, Naturverbundenheit) für überreizte Großstädter konstruiert. Diese Überzeichnungen und diskursiven Zuschreibungen muss eine Kritische Geographie ländlicher Räume adressieren und hinterfragen. Dünckmann erkennt eine „Politik der Idylle“, deren diskursive Produktion politisch und ökonomisch nutzbar wird, um bestimmte Lebensstile zu propagieren oder um das Konstrukt „ländlicher Raum“ diskursiv von sozialen Brüchen oder Widersprüchen zu säubern. In Rückgriff auf Hannah Arendt verweist Dünckmann auf die Gefahr und politische Funktion vermeintlich ,a-politischer ' Ideologien und 
Idyllen, die der Komplexität politischer Debatten mit einer Betonung von Einfachheit und Harmonie begegnen. Mießner/Naumann verweisen auf Beiträge der Genderforschung, die zeigen konnten, dass ländliche Räume oftmals als maskulin besetzte und außerdem als weiße Orte mit ,traditionellen Werten" konstruiert werden, in denen Migration und People of Colour nicht vorkommen. Solche reaktionären Konzepte sind Grundlage für gesellschaftlichen Ausschluss und Rassismus.

Die ungleiche Entwicklung ländlicher Räume vor dem Hintergrund einer politischen Rahmensetzung, in der periphere ländliche Kommunen mit ,wettbewerbsfähigeren" Städten um öffentliche Förderungen (Nahverkehr, Ärzt*innendichte, Schulen etc.) konkurrieren, diskutieren Redepenning/Singer. Die Autoren plädieren allerdings, in Anlehnung an Nancy Frasers Gerechtigkeitsmodel, dafür, raumbezogene Gerechtigkeit nicht nur an einer sozial- und wirtschaftspolitischen Verteilung von Gütern und physischer Ausstattung festzumachen. Ebenso entscheidend sei es, räumliche Exklusionsprozesse durch Anerkennung und Förderung kultureller Diversität zu verhindern, sowie die (politische) Repräsentation ländlicher Kommunen (und aller dort lebenden Bürger*innen) in Entscheidungsprozessen zu gewährleisten. Während ein solches Framing politischer Entscheidungsprozesse innerhalb globalisierter Raumverhältnisse noch fern scheint, lassen sich die Dimensionen raumbezogener Gerechtigkeit als Statuten eines normativ gefüllten ,,inklusiven Place-Making“ durchaus für politische Programmatik nutzen. Dass die Frage gerechter Lebensverhältnisse auch innerhalb einzelner Kommunen gestellt werden muss, verdeutlicht der Beitrag von Kordel/Weidinger/Hachmeister $\mathrm{zu}$ den Lebenswelten geflüchteter Menschen in ländlichen Räumen. So sind einzelne Personengruppen wie geflüchtete Menschen, Frauen, finanzschwache oder körperlich eingeschränkte Personen, Migrant*innen usw. oft von zusätzlicher Exklusion betroffen.

Ein Schwerpunkt des Sammelbands liegt auf den Auswirkungen austeritärer Politik auf Entwicklungen des ländlichen Raums. Kallert/Dudek arbeiten hegemonietheoretisch am Beispiel der bayrischen Landespolitik heraus, wie sich austeritäre Paradigmen als vermeintliches Allgemeininteresse durchsetzen konnten. Die Entschuldungspolitik im Zusammenhang mit dem kommunalen Finanzausgleich, bei gleichzeitiger Aktivierung und Responsibilisierung der Kommunen, fassen sie als „Aktivieren statt Alimentieren“. Nach Kallert/Dudek wird diese Politik über ,economic imaginaries" im Sinne der Cultural-Political-Economy-Analyse durchsetzbar. Die Folgen neoliberaler Politiken kommen auch im letzten Kapitel zu zivilgesellschaftlichem Engagement in ländlichen Räumen verstärkt zur Sprache. So weisen Haunstein als auch Reda in ihrer kritischen Analyse bürgerschaftlicher Verantwortungsübernahme in ländlichperipheren Gebieten darauf hin, dass es gilt, die Debatte um Bürger*inneninitiativen nicht vorschnell auf die Anerkennung deren kreativer Leistung zu reduzieren, sondern statt- dessen ihre Ursache - nämlich die defizitäre Leerstelle, welche aus dem Rückbau staatlicher Daseinsvorsorge und neoliberaler Politik entsteht - anzuvisieren.

Auch widerständige Praktiken blitzen in den Beiträgen auf: So werden Globalisierungs- und Transnationalisierungsprozesse nicht nur von Seiten (über-)staatlicher Institutionen und privatwirtschaftlicher Akteure vorangetrieben, um Interessen des Kapitals durchzusetzen, sondern ebenso von zivilgesellschaftlichen Akteuren genutzt, um lokale Widerständigkeiten zu stützen und zu vernetzen (Brad/Hein). Ein Vorschlag von Jutteau/Authier besteht darin, Henri Lefebvres Konzept des „Droit à la ville“, des „Rechts auf Stadt“, auf ländliche Räume zu übertragen. Im Sinne eines „Recht auf das Dorf" ließe sich dieses durchaus als politische und aktivistische Forderung nach Aneignung ländlicher Räume nutzen, nach einem Recht auf Infrastruktur, Wissen, nach Differenz und Anerkennung aller Personen.

An die existierende Genderforschung innerhalb der Rural Geography knüpft der Beitrag von Oedl-Wieser/Schmitt an. Thematisiert werden ideologische Verschiebungen hin zu einer maskulinen Rechtslastigkeit, die, so die These der Autorinnen, insbesondere in ländlichen Räumen beobachtbar sind. Der von ihnen festgestellte „Urban-Rural-Divide“ bezüglich konservativer und liberaler Lebenskonzepte scheint nicht nur in Anbetracht momentaner Wahlergebnisse hochaktuell. Sie machen auch deutlich, dass es mehr kritische Forschung braucht, die ,geschlechterspezifische Ungleichheiten [...] thematisiert, analysiert und die Komplexität von sozialen, wirtschaftlichen und kulturellen Entwicklungen in ländlichen Räumen erfasst". Da Frauen in politischen Funktionen ländlicher Regionen immer noch stark unterrepräsentiert sind und sich andere bereits thematisierte Probleme (Austerität und Rückbau von Sozialleistungen) verschärfen, drohen Geschlechterverhältnisse ignoriert oder trivialisiert zu werden. Über die soziale Kategorie gender hinaus gilt es, nach Marginalisierung und Exklusionsmechanismen aufgrund von race, ethnicity und class und deren spezifischer Ausprägung in ländlichen Gebieten zu fragen. Hierauf wird innerhalb des Sammelbandes an verschiedenen Stellen verwiesen, empirische Forschungsprojekte sind in der Gesamtschau dennoch etwas unterrepräsentiert.

Von staatstheoretisch-materialistischen, strukturalistischen über poststrukturalistische Ansätze bis hin zur Assemblage-Forschung bieten die Autor*innen eine breit gefächerte Auswahl, die nicht nur Wege für weitere Forschung weist, sondern auch die dringend notwendigen politischen Debatten befeuern könnte. Im Abgleich unterschiedlicher theoretischer und methodischer Forschungsansätze werden Gegensätze, aber auch Schnittpunkte zwischen den verschiedenen Konzeptionen augenfällig. Eine weitere Stärke des Bandes besteht in der Reflexion „blinder Flecken“ der einzelnen Theorien. Viele der Autor*innen kompensieren die konstatierten Leerstellen durch eine Kombination verschiedener Ansätze. 
Den inhaltlich größten Anteil machen dabei Beiträge aus, die sich mit dem Verhältnis von ländlichen Regionen und globalen politischen wie ökonomischen Prozessen beschäftigen. Markus Keck verdeutlicht anhand der Analyse des globalen Agrar- und Ernährungsregimes, wie sich Globalisierungsprozesse in ländliche Räume einschreiben. Er arbeitet aber zugleich heraus, dass diese Prozesse regional äußerst heterogen verlaufen und ausgehandelt werden. Die Analyse polit-ökonomischer Prozesse auf Makroebene mit der Heterogenität und Kleinteiligkeit lokaler Herstellungs- und Aushandlungsprozesse „,des Globalen“ zusammenzubringen, erweist sich als die zentrale Herausforderung, mit denen sich viele Beiträger*innen des Bandes konfrontiert sehen. Keck selbst verwendet einen Ansatz der Assemblage-Forschung, um die Ausgestaltung „eines kleinteiligen Herstellungsprozesses, der mobile Denkmuster, technische Artefakte und konkrete Praktiken umfasst" am Beispiel der Verwendung von Gentechnik in der indischen Agrarwirtschaft greifbar zu machen. Zusätzlich benutzt er Martin Heideggers Begriff des „Weltens“, um damit elitäre Praktiken zu erfassen, die gesellschaftliche Sinnzusammenhänge und Vorstellungswelten erzeugen und durchsetzen.

Einen anderen Zugang wählen Brad/Hein, indem sie aus einer materialistisch-staatstheoretischen Perspektive die Transnationalisierung von Agrarkonflikten am Beispiel Sumatras betrachten. Ausgehend von der Staatstheorie Nicos Poulantzas untersuchen die Autor*innen, wie sich gesellschaftliche Kräfteverhältnisse in staatliche Strukturen einschreiben und staatliche Strategien für den ländlichen Raum beeinflussen, im Fallbeispiel die Förderung der Agrarindustrie zu Lasten des Erhalts kleinbäuerlicher Produktionsweisen. Brad/Hein beschreiben überzeugend und differenziert, wie die Interessen transnationaler Unternehmen durch die strategische Selektivität der Staatsapparate begünstigt werden, sich letztlich materialisieren, aber gleichzeitig lokal ausgehandelt werden müssen.

Auch Nadine Reis nimmt die Auswirkungen zunehmender Finanzialisierung, Exportorientierung und Intensivierung der Landwirtschaft in Räumen der kapitalistischen Peripherie in den Blick und beschreibt, wie sich das Agrobusiness nach dem Vorbild industrieller Großkonzerne organisiert. Immer mehr Menschen aus dem ländlichen Raum werden zu größerer Arbeitsmobilität und in neue Erwerbsmodelle gedrängt. Reis schlussfolgert, dass die Grenzen zwischen ländlichem und städtischem Raum verschwimmen - ein empirischer Befund, der dem vielfach konstatierten „Urban-Rural-Divide“ widerspricht. Zahlreiche der hier vereinten Beispiele verdeutlichen, dass der ländliche Raum in vielen Ländern auBerhalb Europas insbesondere als Produktionsort von Agrargütern und Träger natürlicher Ressourcen charakterisiert ist. Während der Zugang zu Agrarland für viele dort lebende Menschen nach wie vor die Existenz darstellt, konkurrieren sie aber zunehmend mit internationalen Konzernen und Akteuren der Finanzwirtschaft, welche in diesen Flächen Investitionsmöglichkeiten finden - Konflikte um Landnutzungen, veränderte Erwerbsmodelle und tiefgreifende räumliche Rekonfigurationen sind die Folge.

Der Band zeigt, wie bewährte theoretische Ansätze erfolgreich auf Fragen des ländlichen Raums übertragen werden können und liefert zugleich erste Ideen, um entlang inklusiver Place-Konzepte und eines „Rechts auf das Dorf“ Utopien für ländliche Räume zu entwickeln. Da sich viele der angesprochenen Thematiken durch neoliberale Politik- und Wirtschaftsprogramme weiter zu verschärfen drohen, gilt es, die wissenschaftliche wie politische Aufmerksamkeit für ländliche Räume insgesamt deutlich zu erhöhen und in eine selbstkritische, aber zugleich progressive Debatte beider Felder einzusteigen.

Was die Zielsetzung Kritischer Geographie im ländlichen Raum sein könnte, fasst Andrei Dörre pointiert zusammen: Danach müsse die Kritische Geographie positiv wie negativ besetzte Konzepte zur Entwicklung ländlicher Räume kritisch hinterfragen und sich für eine konsequente Sensibilisierung bezüglich existierender Interessenskonflikte, Machtasymmetrien und sozialer Konflikte einsetzen. So kann kritische Forschung hegemoniale Praktiken und Deutungsmuster sichtbar machen sowie politische Konfliktlinien aufzeigen. Zugleich bewahrt sie davor, emanzipatorische Absichten gegen Wunschvorstellungen einzutauschen. Es gilt, und dazu trägt der Band entscheidend bei, mit idealisierenden und verkürzten Darstellungen ländlicher Räume zu brechen und die Politisierung und Entnaturalisierung bestehender gesellschaftlicher Konflikte zu forcieren. 